\title{
A PULSED ELECTRIC LENS FOR NDCX
}

\author{
by \\ E.P. Lee \\ Lawrence Berkeley National Laboratory (on behalf of U.S. HIFS-VNL) \\ 1 Cyclotron Road, Berkeley, CA 94720 ,
}

Accelerator Fusion Research Division

Emest Orlando Lawrence Berkeley National Laboratory

University of Califomia

Berkeley. Califomia 94720

July 2007

This work was supported by the Director, Office of Science, Office of Fusion Energy Sciences, of the U.S. Department of Energy under Contract No. DE-AC02$05 \mathrm{CH} 11231$. 
A pulsed electric tens for NDCX

o

Ear Lee

$\sigma_{u} l y$ 18, 2007

- To compress pulse, $\sqrt{T^{\prime} t}>\sqrt{\text { peed }}$

- This causes a chromatic aberration:

- Place fast Pulsed Lens here "to compensate

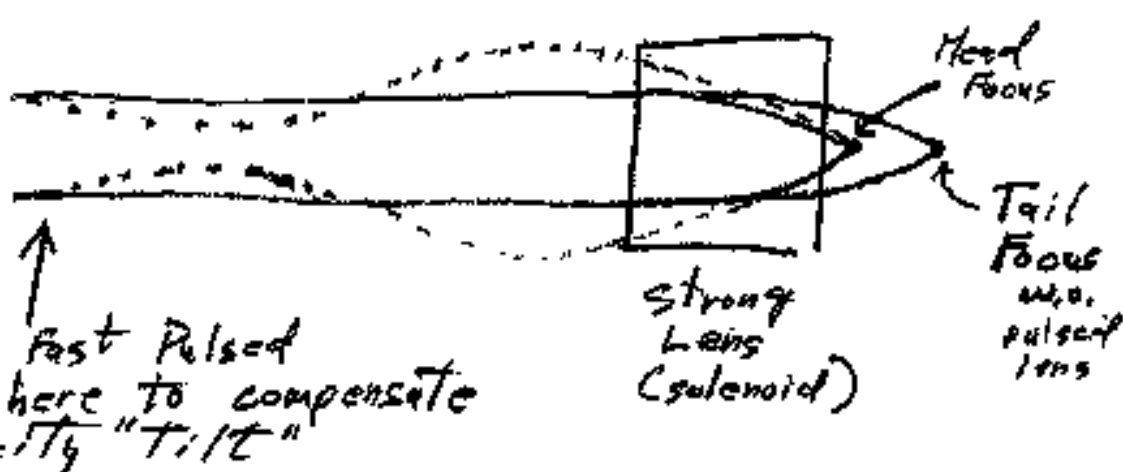

$\Theta$

Considerations

Timon sate is w pulse length w 10 ps

Lens works only in varewum

Lens must be compact (L so om)

Voltages $\approx 100 \mathrm{kV}$

Programeble waveform

Reasonable cost - Hob at energy/power

Solenoid $\Rightarrow 1.0 \in T, 10^{9}$ with

Electric lens $\rightarrow 30 \mathrm{JJ}, 30 \mathrm{~kW}$ 
Axisommetrice multigap lens system

(s)

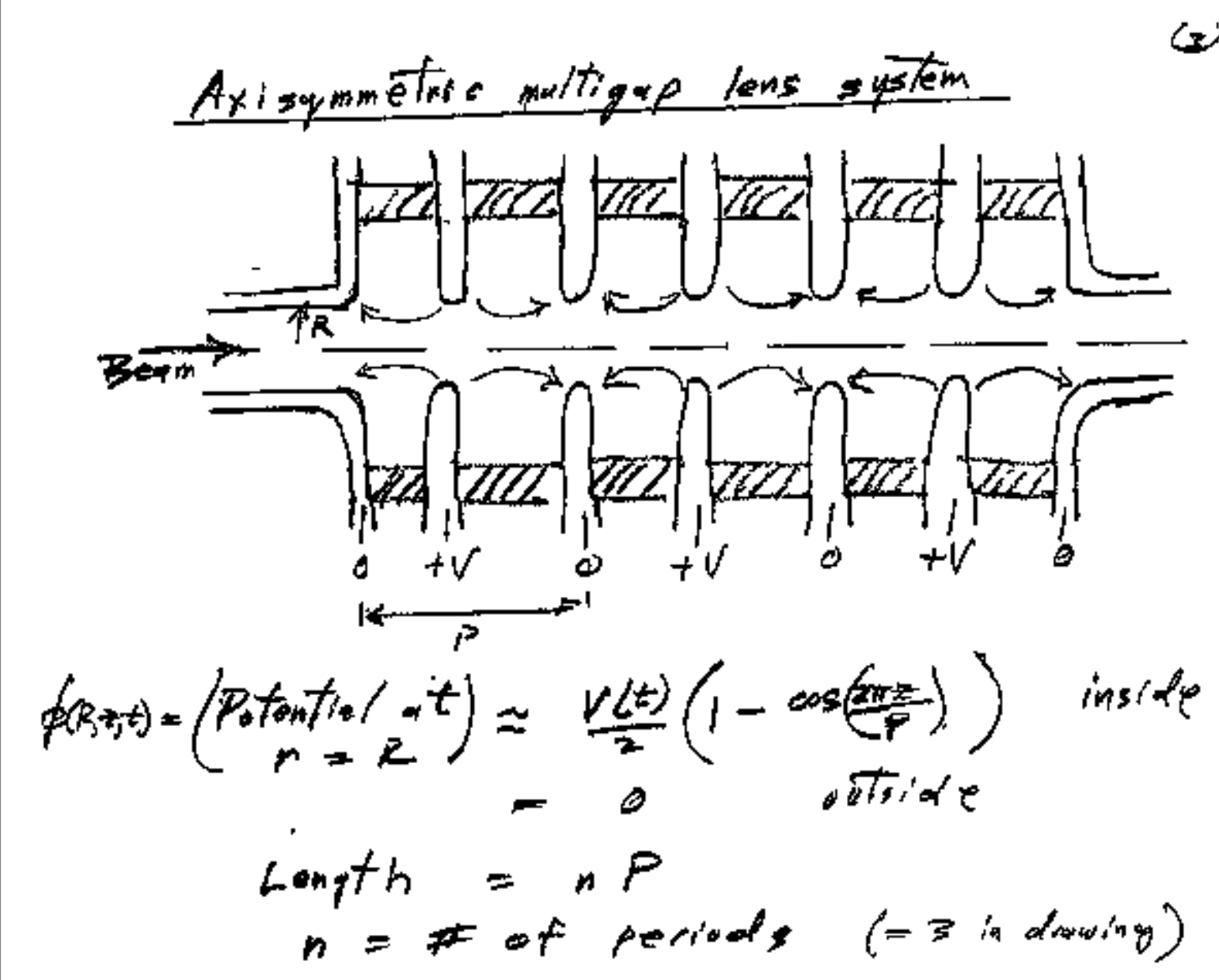

(4)

$$
\begin{aligned}
& \text { Potential inside the bore } C n<R \\
& \nabla^{2} \phi=0 \quad(E=-\vec{\nabla} \phi) \\
& \rightarrow \phi(r, 3, t) \approx \phi(\theta, t)-\frac{2^{2} \phi}{22^{2}} \frac{r^{2}}{4} \\
& \phi_{0}(z, t)=\text { on-axis potential }
\end{aligned}
$$

$\therefore$ Solve $\left.\frac{\partial^{2} \phi_{0}}{\partial T^{2}}-\frac{4}{R^{2}} \phi_{0}=-\frac{y}{R^{2}} \phi(R, \theta), t\right)$

$$
\begin{aligned}
& =-\frac{4}{R^{2}} \frac{V(\alpha-1)}{2}\left(1-\cos \left(\frac{\pi \pi^{2}}{p}\right)\right) \text { (intis) }
\end{aligned}
$$

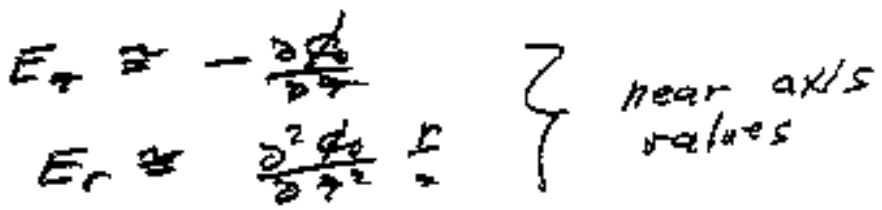

- This ann be dane analytically - 
Solve for lon orbits

(5)

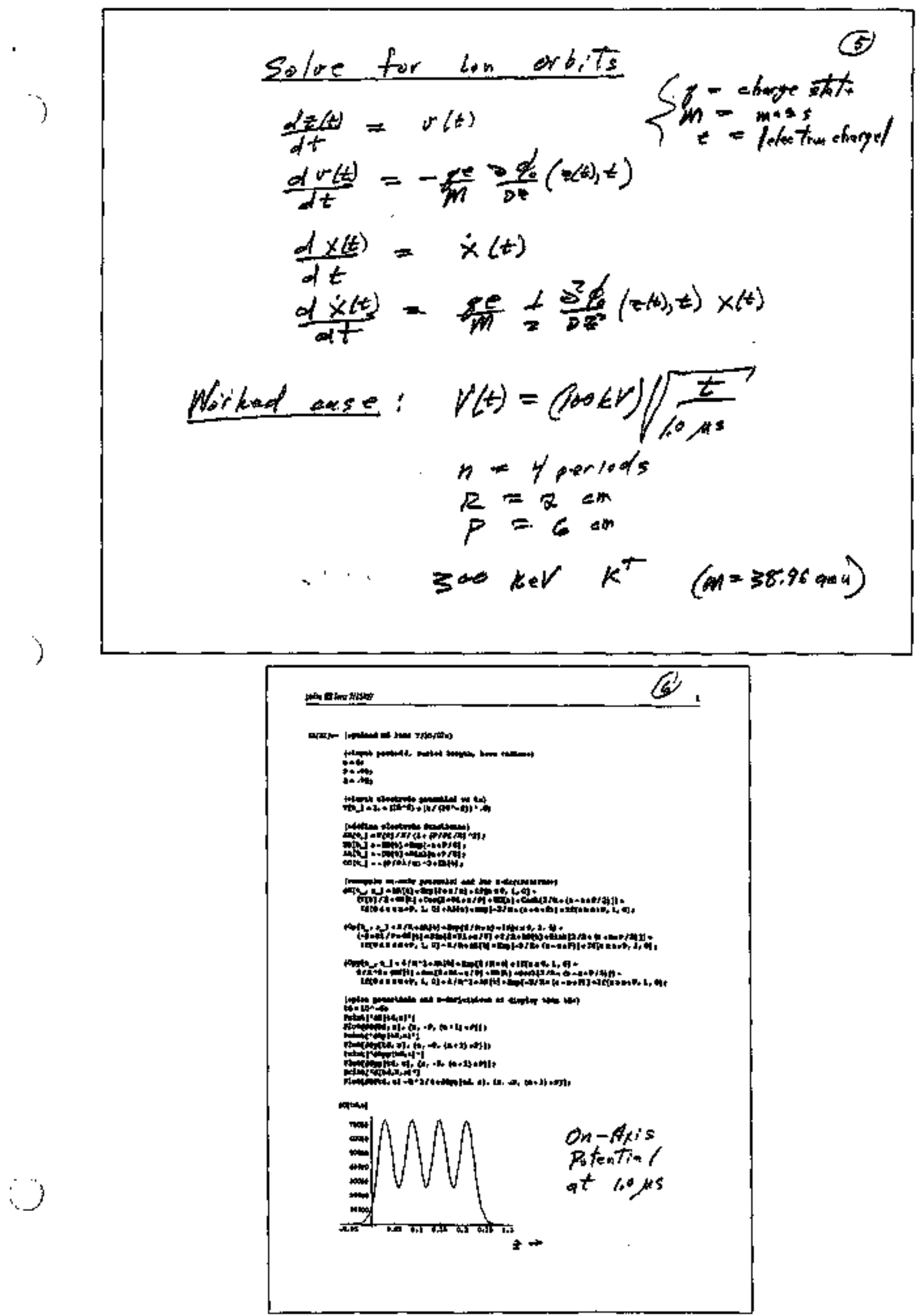



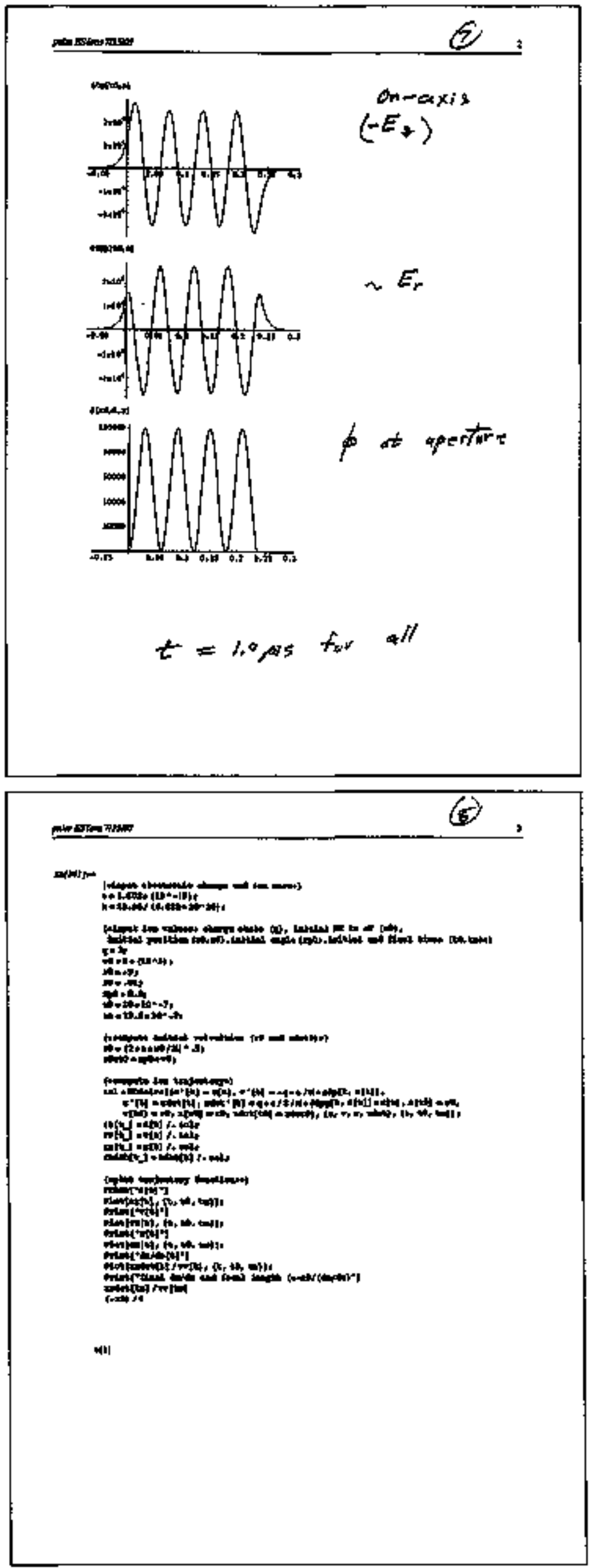

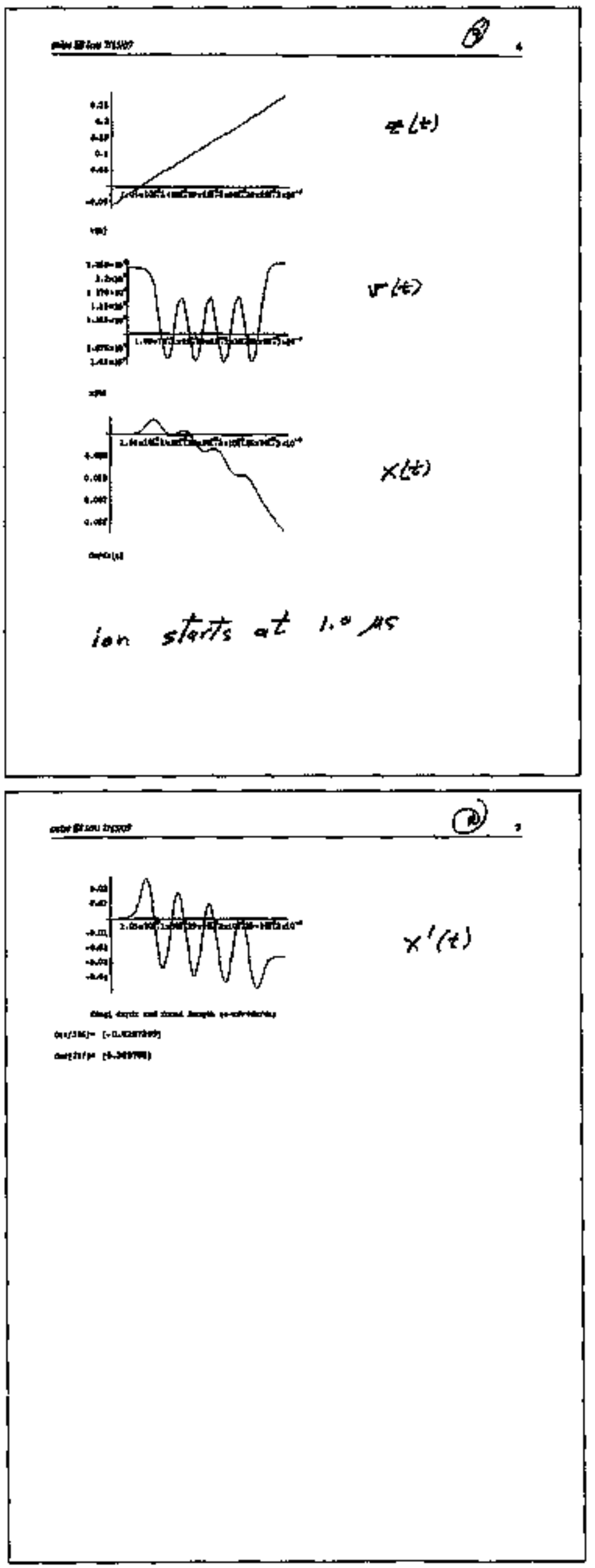
To find foed lempth start lons $]^{\text {(ii) }}$ at warions tines with $x=10 \mathrm{~cm}$, 楚 $=0$.

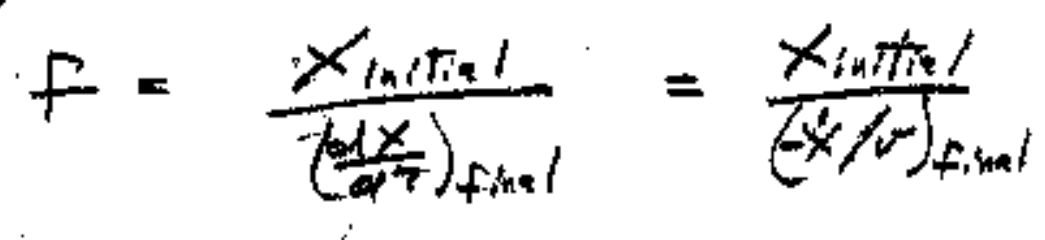

\begin{tabular}{|c|c|c|}
\hline 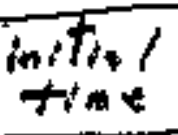 & $\begin{array}{l}\text { fin } 1 \\
\text { dx/ur }\end{array}$ & fore $/$ th \\
\hline $\begin{array}{l}0 \\
.2 \mu s \\
.4 \\
.8 \\
8 \\
1.8\end{array}$ & $\begin{array}{l}-.00321 \\
-.00716 \\
-.01167 \\
-.0103 \\
-.0210 \\
-.0257\end{array}$ & $\begin{array}{l}7.12 \mathrm{~m} \\
1.10 \\
.860 \\
.615 \\
.477 \\
.389\end{array}$ \\
\hline
\end{tabular}

